\title{
Brain-Derived Neurotrophic Factor Is Associated with Age-Related Decline in Hippocampal Volume
}

\author{
Kirk I. Erickson, ${ }^{1}$ Ruchika Shaurya Prakash, ${ }^{2}$ Michelle W. Voss, ${ }^{3}$ Laura Chaddock, ${ }^{3}$ Susie Heo, ${ }^{3}$ Molly McLaren, ${ }^{1}$ \\ Brandt D. Pence, ${ }^{4}$ Stephen A. Martin, ${ }^{4}$ Victoria J. Vieira, ${ }^{4}$ Jeffrey A. Woods, ${ }^{4}$ Edward McAuley, ${ }^{4}$ and Arthur F. Kramer ${ }^{3}$ \\ ${ }^{1}$ Department of Psychology, University of Pittsburgh, Pittsburgh, Pennsylvania 15260, ${ }^{2}$ Department of Psychology, The Ohio State University, Columbus, \\ Ohio 43210, and ${ }^{3}$ Department of Psychology, The Beckman Institute for Advanced Science and Technology and ${ }^{4}$ Department of Kinesiology and \\ Community Health, University of Illinois, Urbana, Illinois 61801
}

Hippocampal volume shrinks in late adulthood, but the neuromolecular factors that trigger hippocampal decay in aging humans remains a matter of speculation. In rodents, brain-derived neurotrophic factor (BDNF) promotes the growth and proliferation of cells in the hippocampus and is important in long-term potentiation and memory formation. In humans, circulating levels of BDNF decline with advancing age, and a genetic polymorphism for BDNF has been related to gray matter volume loss in old age. In this study, we tested whether age-related reductions in serum levels of BDNF would be related to shrinkage of the hippocampus and memory deficits in older adults. Hippocampal volume was acquired by automated segmentation of magnetic resonance images in 142 older adults without dementia. The caudate nucleus was also segmented and examined in relation to levels of serum BDNF. Spatial memory was tested using a paradigm in which memory load was parametrically increased. We found that increasing age was associated with smaller hippocampal volumes, reduced levels of serum BDNF, and poorer memory performance. Lower levels of BDNF were associated with smaller hippocampi and poorer memory, even when controlling for the variation related to age. In an exploratory mediation analysis, hippocampal volume mediated the age-related decline in spatial memory and BDNF mediated the age-related decline in hippocampal volume. Caudate nucleus volume was unrelated to BDNF levels or spatial memory performance. Our results identify serum BDNF as a significant factor related to hippocampal shrinkage and memory decline in late adulthood.

\section{Introduction}

Significant shrinkage of gray matter occurs in late adulthood, with the hippocampus showing disproportionately faster rates of decay than sensory areas (Raz et al., 2005; Driscoll et al., 2009; Kennedy et al., 2009). The prefrontal cortex, entorhinal cortex, and caudate nucleus also shrink in late adulthood and contribute to the pattern of cognitive decline often observed in older adults (Raz et al., 2005; Driscoll et al., 2009; Walhovd et al., 2009). However, despite the convincing pattern of regional brain shrinkage, very little is known about the underlying molecular mechanisms that provoke deterioration of brain tissue in elderly humans (Fjell et al., 2010). Given the regionally specific patterns of age-related deterioration and regional variation in the concentration of molecules that could be contributing to volume decline, it is unlikely that a common molecular factor would explain age-related shrinkage of brain tissue in all regions. Instead, it is more likely that the molecular mechanisms underlying hippocampal shrinkage are different from those underlying shrinkage of the prefrontal cortex or caudate nucleus (Fjell et al., 2010).

Received Dec. 17, 2009; revised March 1, 2010; accepted March 5, 2010.

This work was supported by National Institute on Aging Grants R01 AG25667 and R01 AG25302. We thank the following people for their assistance during data collection: Susan Herrel, Nancy Dodge, Holly Tracy, Dawn Epstein, Zuha Warraich, Jennifer Kim, Maritza Alvarado, Heloisa Alves, Edward Malkowski, and Jason Lewis.

Correspondence should be addressed to Dr. Kirk I. Erickson, Department of Psychology, University of Pittsburgh, 3107 Sennott Square, 210 S. Bouquet Street, Pittsburgh, PA 15260. E-mail: kiericks@pitt.edu.

DOI:10.1523/JNEUROSCI.6251-09.2010

Copyright $\odot 2010$ the authors $\quad 0270-6474 / 10 / 305368-08 \$ 15.00 / 0$
Changes in the concentration of brain-derived neurotrophic factor (BDNF) might be contributing to shrinkage of the hippocampus in late adulthood. BDNF, a molecule that is highly concentrated in the hippocampus (Phillips et al., 1990; Wetmore et al., 1990; Murer et al., 2001), is important in synaptic plasticity (Kang and Schuman, 1995; Figurov et al., 1996; Stoop and Poo, 1996; Pang et al., 2004; Tanaka et al., 2008) and is thought to contribute to neurogenesis in the dentate gyrus (Takahashi et al., 1999; Benraiss et al., 2001; Pencea et al., 2001), but its concentration declines in late adulthood (Lommatzsch et al., 2005; Ziegenhorn et al., 2007) (but see Lapchak et al., 1993). In humans, a single nucleotide polymorphism in the BDNF gene affects the regulated secretion of BDNF in the hippocampus (Egan et al., 2003) and has been related to lower serum levels of BDNF (Ozan et al., 2010) and smaller hippocampal volumes (Pezawas et al., 2004; Szeszko et al., 2005; Bueller et al., 2006). Smaller hippocampal volumes predict more rapid conversion to dementia (Grundman et al., 2002) and poorer memory function (Erickson et al., 2009).

BDNF is also present in the caudate nucleus but is less concentrated than in the hippocampus (Altar et al., 1993; Kawamoto et al., 1996; Schmidt-Kastner et al., 1996; Murer et al., 2001). Therefore, we reasoned that declining levels of BDNF might have regionally specific effects and explain age-related loss of volume in the hippocampus but not the caudate nucleus. Furthermore, both the hippocampus and caudate nucleus are involved in learning and memory: the caudate nucleus with procedural memory and the hippocampus with episodic memory. Therefore, we pre- 


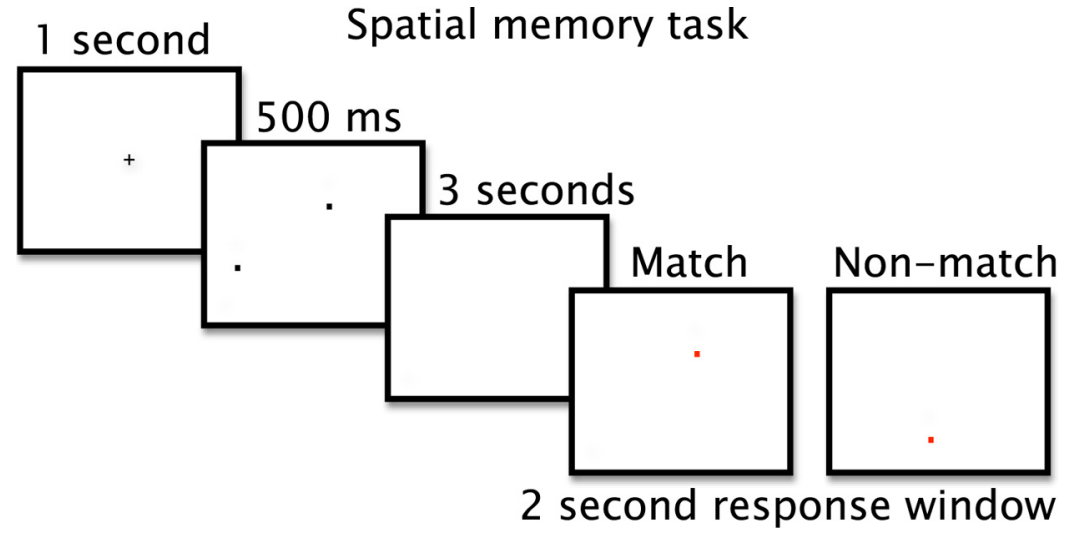

Figure 1. The spatial memory task load was parametrically manipulated between one, two, or three items (2-item condition shown here). Participants were asked to remember the locations of one, two, or three black dots. After a brief delay, a red dot appeared, and participants were asked to respond whether the location of the red dot matched or did not match one of the locations of the previously shown black dots.

dicted that age-related loss of hippocampal volume, but not caudate nucleus volume, would translate to poor memory performance on a task related to hippocampal function (Erickson et al., 2009) and that serum BDNF levels would be related to decline in hippocampal volume and memory performance.

\section{Materials and Methods}

Participants. We recruited 142 participants between 59 and 81 years of age (mean, 66.5 years; $76 \%$ female). All participants were screened for dementia with the revised and modified Mini-Mental Status Examination (Kukull et al., 1994) and were excluded from participation if they did not reach the required cutoff of 51 (maximum score of 57). All participants met or surpassed criteria for participating in a magnetic resonance imaging (MRI) study, including no previous head trauma, no previous head or neck surgery, no diagnosis of diabetes, no neuropsychiatric or neurological condition including brain tumors, and no metallic implants that could interfere with or cause injury attributable to the magnetic field. Finally, all participants signed an informed consent approved by the University of Illinois.

MRI protocol and image processing. For all participants, highresolution $(1.3 \times 1.3 \times 1.3 \mathrm{~mm})$ T1-weighted brain images were acquired using a three-dimensional magnetization prepared rapid gradient echo imaging protocol with 144 contiguous slices collected in an ascending fashion. All images were collected on a $3 \mathrm{~T}$ Siemens Allegra scanner with an echo time of $3.87 \mathrm{~ms}$, repetition time of $1800 \mathrm{~ms}$, field of view of $256 \mathrm{~mm}$, an acquisition matrix of $192 \times 192 \mathrm{~mm}$, and a flip angle of $8^{\circ}$.

For segmentation and volumetric analysis of the left and right hippocampus and caudate nucleus, we used the Oxford University Centre for Functional MRI of the Brain (FMRIB) Integrated Registration and Segmentation Tool (FIRST) in the FMRIB Software Library (FSL) version 4.0. FIRST is a semiautomated model-based subcortical segmentation tool using a Bayesian framework from shape and appearance models obtained from manually segmented images from the Center for Morphometric Analysis, Massachusetts General Hospital (Boston, MA). Structural and landmark information were obtained from 317 manually segmented and labeled T1-weighted images of the brain from normal children, adults, and pathological populations (including schizophrenia and Alzheimer's disease) and were modeled as a point distribution model in which the geometry and variation of the shape of the structure are submitted as priors. Volumetric labels are parameterized by a threedimensional deformation of a surface model based on multivariate Gaussian assumptions. FIRST then searches through linear combinations of shape modes of variation for the most probable shape given the intensity distribution in the T1-weighted image (for an additional description of this method, see Patenaude et al., 2007a,b). Previous studies have successfully used this technique to segment hippocampal volumes in elderly individuals (Erickson et al., 2009, 2010).
This method first runs a two-stage affine registration to a standard space template (Montreal Neurological Institute space) with 1 $\mathrm{mm}$ resolution using 12 degrees of freedom and a subcortical mask to exclude voxels outside the subcortical regions. Second, the left and right hippocampus and caudate nucleus were segmented with 30 modes of variation. Modes of variation were optimized based on leave-one-out cross-validation on the training set and increases the robustness and reliability of the results (Patenaude et al., 2007b). Finally, boundary correction takes place for each structure that classifies the boundary voxels as belonging to the structure or not based on a statistical probability $(Z$ score $>3.00 ; p<0.001)$. The hippocampus volume comprised the dentate gyrus, the ammonic subfields (CA1-CA4), the prosubiculum, and the subiculum. The caudate nucleus comprised both the head and tail of the region. Segmentations from each participant were visibly checked for any significant error that could have occurred during the segmentation process. No errors were noted.

Intracranial volume (ICV) is frequently used to adjust the regional volumes for gender and for height (Raz et al., 2005). Here, we calculated ICV as the sum of gray, white, and CSF and adjusted the volume of each region by this measure using the FMRIB automated segmentation tool in FSL version 4.0 (Zhang et al., 2001; Smith et al., 2004). In accordance with other volumetric analyses, adjustment was performed for each region by an analysis of covariance approach: adjusted volume $=$ raw volume $-b \times(\mathrm{ICV}-$ mean ICV), where $b$ is the slope of a regression of a region of interest volume on ICV (Raz et al., 2005; Erickson et al., 2009; Kennedy et al., 2009). Adjusted volume was used for all analyses described in this study.

Spatial memory task. To test whether BDNF and hippocampal volume would be related to age-related changes in memory function, all participants completed a computerized spatial memory task approximately 1 week before the MR session. Spatial memory was tested by a task associated with a genetic risk for Alzheimer's disease and hippocampal volume (Greenwood et al., 2005; Erickson et al., 2009). First, a fixation crosshair appeared for $1 \mathrm{~s}$, and participants were instructed to keep their eyes on the crosshair. After the fixation, one, two, or three black dots appeared at random locations on the screen for $500 \mathrm{~ms}$. The dots were removed from the display for a period of $3 \mathrm{~s}$. During this time, participants were instructed to try to remember the locations of the previously presented black dots. At the end of the $3 \mathrm{~s}$ delay, a red dot appeared on the screen in either one of the same locations as the target dots (match condition) or at a different location (nonmatch condition). Participants had $2 \mathrm{~s}$ to respond to the red dot by pressing one of two keys on a standard keyboard: the " $\mathrm{x}$ " key for a nonmatch trial, and the "m" key for a match trial (Fig. 1). Forty trials were presented for each set size (one, two, or three locations), with 20 trials as match trials and 20 trials as nonmatch trials. Participants were instructed to respond as quickly and accurately as possible. Several practice trials were performed before the task began to acquaint the participants with the task instructions and responses.

Blood collection and BDNF ELISA. Blood sampling for BDNF analysis was performed approximately 2 weeks before the MRI session. Fasted subjects reported to the laboratory at 8:00 A.M., at which time blood from the antecubital vein was collected in sterile serum separator tubes (BD Biosciences). The blood samples were kept at room temperature for $15 \mathrm{~min}$ to allow for clotting, after which the samples were centrifuged at $1100 \times g$ at $4^{\circ} \mathrm{C}$ for $15 \mathrm{~min}$. Serum was then harvested, aliquoted, and stored at $-80^{\circ} \mathrm{C}$ until analysis. Serum BDNF was quantified using an ELISA (ChemiKine BDNF Sandwich ELISA kit, CYT306; Millipore Bioscience Research Reagents) following the instructions of the manufacturer. The intra-assay and interassay coefficients of variation were 3.7 and $8.5 \%$, respectively. Briefly, serum samples were diluted 1:80 in the supplied sample diluent and assayed against a standard curve with a 500 
$\mathrm{pg} / \mathrm{ml}$ highest concentration. The supplied mouse anti-human BDNF-biotin primary antibody and streptavidin-horseradish peroxidase secondary antibody were used at a dilution factor of 1:1000. After incubation with the provided substrate solution, the reaction was stopped with the addition of stop solution, and the plate was read at $450 \mathrm{~nm}$ using a spectrophotometric plate reader (Multiskan Plus; Thermo Labsystems).

Analyses. We examined whether age and BDNF levels were related to volume of the hippocampus or caudate nucleus by a series of multiple regression analyses with sex entered as a control variable. Previous studies and metaanalyses have found that sex covaries with BDNF (Fukumoto et al., 2010). Because this study focused on BDNF associations with aging, we wanted to isolate and remove any variation related to sex. However, we also examined any moderating effects of sex on hippocampal volume and memory performance by running additional analyses with interaction terms included in the model. We report $T$ scores and standardized $\beta$ values. Age and BDNF levels were entered as continuous regressors with left and right hippocampal volume, left and right caudate nucleus volume, and spatial memory performance [accuracy rates (percentage correct) and response times] entered as dependent variables in separate multiple regression models. Normality and homoscedasticity of the residuals were interrogated using $Q-Q$ and $P-P$ plots. All correlation coefficients from the $Q-Q$ plots were linear $\left(r_{\mathrm{q}}>\right.$ $0.99)$, suggesting that all residuals followed a unit normal distribution without outliers. We used Shapiro-Wilk statistics to confirm normality of the residuals $(W>0.987)$. We can conclude from these diagnostic tests that our data were suitable for multiple linear regression analyses. To correct for multiple comparisons, we used a false discovery rate of $p<$ 0.026 as a threshold for all results from the regression analyses.

As an exploratory analysis, we examined whether (1) hippocampal volume would mediate spatial memory decline, (2) BDNF would mediate hippocampal decay, and (3) BDNF would mediate spatial memory deficits (Fig. $2 B$ ). Detailed descriptions of mediation analyses have been described in depth previously (Salthouse et al., 2003; Madden et al., 2007; Erickson et al., 2009). Briefly, mediation is a hypothesis about causal relations, but conclusions of a causal relation are only valid if the assumptions are valid (Judd and Kenny, 1981; Baron and Kenny, 1986; MacKinnon et al., 2007). Mediation analyses can be conducted by running a series of multiple regression analyses such as those described above. If a relation exists between an independent variable $(A)$ and a dependent variable $(B)$, a third variable might mediate the relation between $A$ and $B$ if controlling for the variance attributable to the mediator variable reliably reduces the variance in $B$ explained by $A$.

The hippocampus volume, but not the caudate nucleus, met criteria for a test of mediation (see Results). To test whether BDNF mediates age-related decline in hippocampal volume, we first ran regressions between age and hippocampal volume with sex entered as a control variable. This allowed us to determine the relation between age and hippocampal volume after adjusting for variation attributable to sex. Next, a regression analysis was conducted between BDNF and left and right hippocampal volume with sex entered as a control variable. This allowed us to determine the relation between BDNF and hippocampal volume. Finally, a regression analysis was conducted with sex entered as a control variable, and BDNF and age entered as continuous variables of interest. If the magnitude of the association between age and hippocampal volume is significantly reduced when BDNF levels are controlled, then it can be concluded that BDNF levels mediate the relation between age and hippocampal volume. Similar tests were done to examine whether BDNF or hippocampal volume mediates the relation between age and memory deficits. To test whether the effect of mediation was significant, we used a version of the Sobel test (Sobel, 1982) popularized by Baron and Kenny (1986). The Sobel test determines whether the effect of the mediator on the dependent variable is significantly different from zero using a two-tailed $z$ test with \pm 1.96 as the critical values in a unit normal distribution. Simulations suggest that the modified Sobel test is preferable when sample sizes are larger than 50 (MacKinnon et al., 1995).

\section{Results}

Hippocampal, but not caudate nucleus, volume declines with increasing age

Consistent with previous research (Raz et al., 2005), hippocampal volume declined with advancing age after adjusting for total intracranial volume and sex (Fig. 3). Multiple regression analyses indicated a main effect of age on the left hippocampus $(\beta=$ $-0.37 ; T=-5.38 ; p<0.001)$ and the right hippocampus $(\beta=$ $-0.40 ; T=-5.92 ; p<0.001)$. However, inconsistent with some previous studies (Raz et al., 2005), we failed to find an age-related decline in volume of the left caudate nucleus $(\beta=0.03 ; T=0.37$; NS) or the right caudate nucleus $(\beta=-0.02 ; T=0.30 ; \mathrm{NS})$.

\section{BDNF levels decline with increasing age}

Consistent with other studies (Lommatzsch et al., 2005; Ziegenhorn et al., 2007), serum BDNF levels declined with advancing age (Fig. $2 A)$. Specifically, we found that, after adjusting for variation attributable to sex, increasing age was negatively associated with BDNF levels $(\beta=-0.24 ; T=-3.20 ; p<0.002)$.

\section{Mediation effects of BDNF and age-related decline in hippocampal volume}

As described in Materials and Methods, mediation is a statistical method to test causal associations (MacKinnon et al., 2007). However, the interpretations of causality in a mediation analysis are only valid as long as the assumptions of the direction of the effects between variables are valid and all other potentially confounding variables are accounted for. In the exploratory analysis and model we propose here, we make the assumption that agerelated changes in BDNF precipitate hippocampal deterioration and not that hippocampal deterioration would precipitate a decline in circulating BDNF levels (Fig. $2 \mathrm{~B}$ ). The latter hypothesis, however, is feasible and should be considered when interpreting the results herein (see Discussion).

First, levels of BDNF were positively correlated with left $(\beta=0.19 ; T=2.52 ; p<0.01)$ and marginally with the right $(\beta=0.14 ; T=1.89 ; p<0.06)$ hippocampal volumes (Fig. 4 ). Furthermore, BDNF levels mediated the relation between increasing age and shrinkage of hippocampal volume. Specifically, BDNF mediated the age-related decline in left hip- 


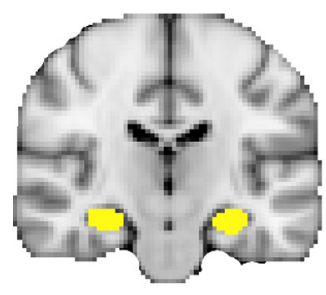

A

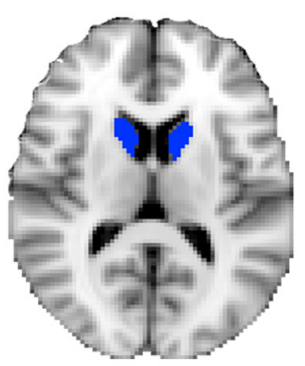

B
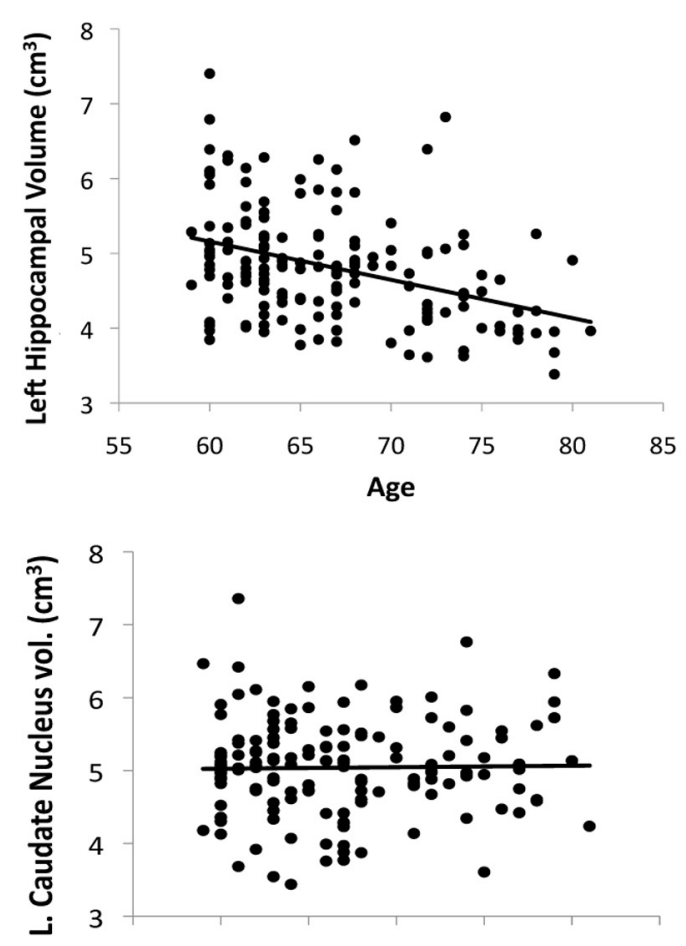

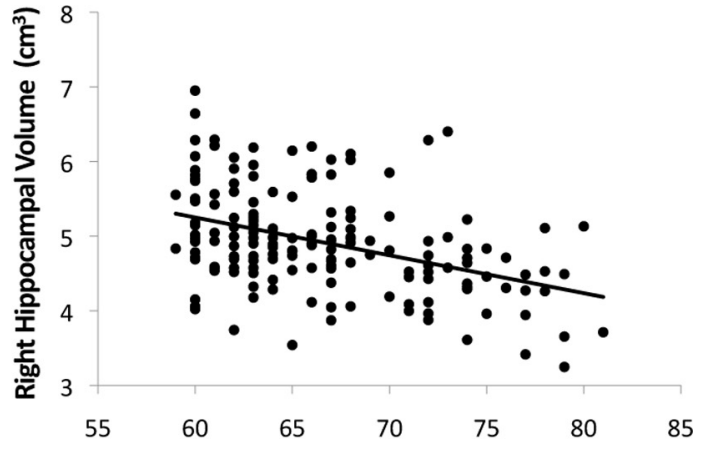

Age

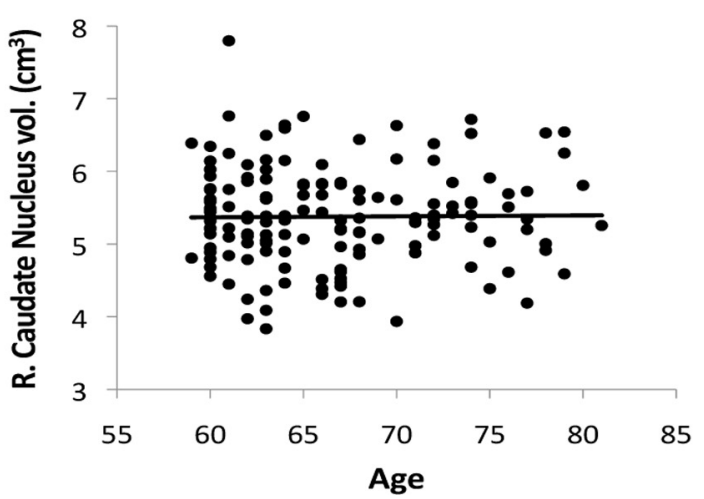

Figure 3. A, Example of hippocampal segmentation and scatter plots showing decline in volume of the left and right hippocampus between 59 and 81 years of age (significant at $p<0.001$ ). $\boldsymbol{B}$, Example of caudate nucleus segmentation and scatter plots showing no significant decline in volume of the caudate nucleus between 59 and 81 years of age.
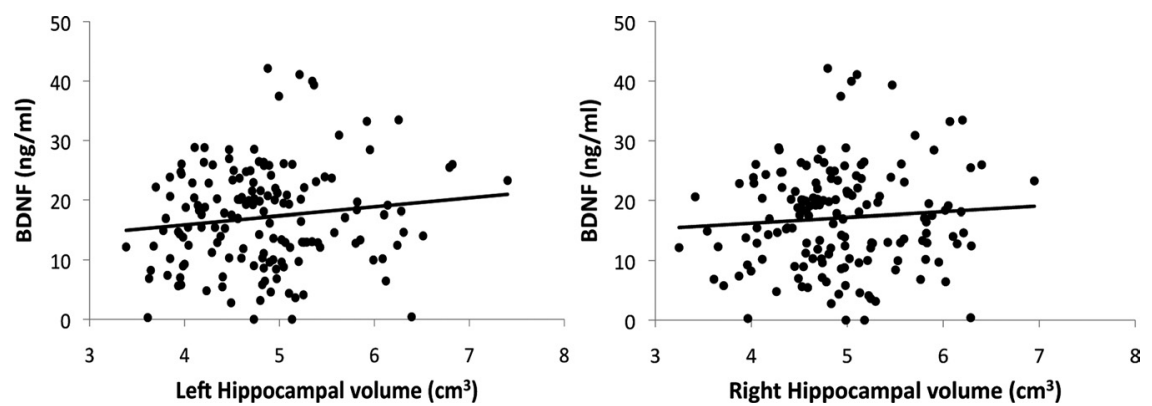

Figure 4. Scatter plots of the association between the volume of the left and right hippocampus and BDNF levels (left, significant at $p<0.05$; right, marginally significant at $p<0.06$ ).

pocampal volume $(Z=-2.13 ; p<0.03)$ and marginally with the right hippocampal volume $(Z=-1.87$; $p<0.06)$. After including BDNF in the regression model, the effect of age on the left hippocampal volume was attenuated but still significant $(\beta=$ $-0.32 ; T=-4.32 ; p<0.001)$, as was the effect of age on right hippocampal volume $(\beta=-0.37 ; T=-5.17 ; p<0.001)$. This finding suggests that an age-related decline in BDNF levels partially contributes to the volumetric shrinkage of the hippocampus associated with advancing age.

BDNF is unrelated to caudate nucleus volume

We tested whether caudate nucleus volume was correlated with BDNF levels after adjusting for sex. We found that BDNF was not predictive of either the left caudate nucleus volume ( $\beta=0.04 ; T=0.52 ; \mathrm{NS})$ or the right caudate nucleus volume $(\beta=0.04 ; T=0.55 ; \mathrm{NS})$.
Spatial memory performance declines with increasing age

We found that memory performance declined with advancing age (Table 1). Specifically, after adjusting for sex, increasing age was associated with lower accuracy rates for the one-item condition $(\beta=$ $-0.23 ; T=-2.98 ; p<0.003)$, the twoitem condition $(\beta=-0.19 ; T=-2.54$; $p<0.01)$, and the three-item condition $(\beta=-0.35 ; T=-4.67 ; p<0.001)$ of the spatial memory task. Similarly, response times increased with advancing age for the one-item condition $(\beta=0.17 ; T=2.28$; $p<0.02)$, the two-item condition $(\beta=$ $0.20 ; T=2.76 ; p<0.006)$, and the threeitem condition $(\beta=0.18 ; T=2.40 ; p<0.01)$.

Mediation effects between hippocampal volume and age-related decline in spatial memory performance Larger hippocampal volumes were positively associated with spatial memory performance (Table 1). Specifically, left hippocampus volume was positively related to accuracy rates for the one-item condition $(\beta=0.26 ; T=3.30 ; p<0.001)$, the two-item condition $(\beta=0.22 ; T=2.75 ; p<0.007)$, and the three-item condition $(\beta=0.35 ; T=4.52 ; p<0.001)$, and right hippocampus volume was positively related to accuracy rates for the one-item condition $(\beta=0.23 ; T=2.82 ; p<0.005)$, the two-item condition $(\beta=0.37 ; T=-5.38 ; p<0.001)$, and the three-item condition $(\beta=0.19 ; T=2.40 ; p<0.01)$. Similar effects were found between the left and right hippocampus and response times (Table 1). Because of the covariation between age and hippocampal volume, we examined whether hippocampal volume 
Table 1. Partial correlations (controlling for sex) between measures of memory performance and age, BDNF, the left hippocampus volume, the right hippocampus volume, the left caudate nucleus volume, and the right caudate nucleus volume

\begin{tabular}{|c|c|c|c|c|c|c|}
\hline & One-item RT & Two-item RT & Three-item RT & One-item ACC & Two-item ACC & Three-item ACC \\
\hline Age & $0.21^{* *}$ & $0.24^{* *}$ & $0.22^{* *}$ & $-0.26^{* *}$ & $-0.21^{* *}$ & $-0.38^{* * *}$ \\
\hline BDNF & $-0.22^{* *}$ & $-0.16^{*}$ & $-0.22^{* *}$ & 0.05 & 0.12 & 0.06 \\
\hline Left hippocampus & $-0.33^{* * *}$ & $-0.25^{* *}$ & $-0.22^{* *}$ & $0.23^{* *}$ & $0.17^{*}$ & $0.33^{* * *}$ \\
\hline Right hippocampus & $-0.24^{* *}$ & $-0.20^{* *}$ & $-0.16^{*}$ & $0.20^{* *}$ & 0.14 & $0.30^{* * *}$ \\
\hline Left caudate nucleus & 0.03 & 0.05 & 0.10 & 0.07 & 0.03 & 0.03 \\
\hline Right caudate nucleus & 0.02 & 0.03 & 0.09 & -0.02 & -0.003 & 0.006 \\
\hline
\end{tabular}

${ }^{*} p<0.05 ;{ }^{* *} p<0.01 ;{ }^{* * *} p<0.001$. RT, Response time; ACC, accuracy.

was related to spatial memory performance even after adjusting for variation attributable to age. When age was included as a covariate in the regression model, the associations between hippocampal volume and spatial memory performance described above remained significant (all $p<0.05$ ).

We ran a series of mediation analyses to determine whether hippocampal volume significantly mediated age-related deficits for any condition on the spatial memory task. In short, we found that volume of the left and right hippocampus mediated agerelated changes in performance for some of the spatial memory conditions. We found that the left hippocampus significantly mediated the age-related increase in response times for the one-item condition $(Z=3.26 ; p<0.001)$ and the two-item condition $(Z=$ 2.38; $p<0.01)$ and marginally for the three-item condition $(Z=$ $1.86 ; p<0.06)$. The left hippocampus also mediated age-related deficits in accuracy rates for the one-item condition $(Z=-2.20$; $p<0.02)$ and the three-item condition $(Z=-2.59 ; p<0.009)$ and marginally for the two-item condition $(Z=-1.83$; $p<$ $0.06)$. The volume of the right hippocampus was less robustly related to age-related memory loss. Specifically, the volume of the right hippocampus significantly mediated the age-related increase in response times for the one-item condition $(Z=2.55$; $p<0.01)$ and marginally for the two-item condition $(Z=1.76$; $p<0.07)$ but failed to mediate the age-related increase in response times for the three-item condition $(Z=1.23$; $p<0.21)$. The volume of the right hippocampus marginally mediated the age-related decline in accuracy rates for the one-item condition $(Z=-1.69 ; p<0.08)$, failed to mediate the age-related decline in accuracy for the two-item condition $(Z=-1.45 ; p<0.14)$, but significantly mediated the age-related decline in accuracy rates for the three-item condition $(Z=-2.47 ; p<0.01)$. It is evident from these results that the volume of the left hippocampus mediates age-related declines in performance on this task more than the right hippocampus.

\section{Caudate nucleus volume is unrelated to spatial memory performance}

As expected, there were no significant associations between caudate nucleus volume and spatial memory function (Table 1). In a series of multiple regression analyses, we found that the left caudate nucleus was not significantly related to accuracy rates for the one-item condition $(\beta=0.09 ; T=1.13$; NS), the two-item condition $(\beta=0.07 ; T=0.84 ; \mathrm{NS})$, or the three-item condition $(\beta=$ 0.06 ; $\mathrm{T}=0.77$; NS). Similarly, the right caudate nucleus volume was not significantly related to accuracy rates for the one-item $(\beta=-0.00 ; T=-0.03 ; \mathrm{NS})$, the two-item $(\beta=0.03 ; T=0.45$; NS), or the three-item $(\beta=0.03 ; T=0.42$; NS) memory conditions. Response times for all three memory conditions were also unrelated (all $p>0.05$ ) to left or right caudate nucleus volume (Table 1). Because of the lack of an association between memory performance and caudate nucleus volumes, mediation analyses were not undertaken with these data.
BDNF is positively related to spatial memory performance BDNF levels were negatively associated with response times on the spatial memory task after controlling for sex (Table 1). Specifically, higher levels of BDNF were associated with faster responding on the one-item memory condition $(\beta=-0.22 ; T=$ $-2.75 ; p<0.007)$, the two-item memory condition $(\beta=-0.17$; $T=-2.19 ; p<0.03)$, and the three-item condition $(\beta=-0.23$; $T=-2.88 ; p<0.005)$. Importantly, even after adjusting for age, the association between BDNF and memory performance measured by response times as described above remained significant (all $p<$ 0.05). Interactions with sex were not significant. Conversely, BDNF was unrelated to accuracy rates. BDNF was unrelated to accuracy for the one-item condition $(\beta=0.04 ; T=0.46$; NS), the two-item condition $(\beta=0.11 ; T=1.34$; NS), and the three-item condition $(\beta=0.04 ; T=0.56 ; \mathrm{NS})$. After including age in the regression model along with interactions with sex, the relationship between BDNF and accuracy rates remained nonsignificant (all $p>0.05$ ).

In a mediation analysis, we found that BDNF significantly mediated the age-related increase in response times for the one-item condition $(Z=1.96 ; p<0.05)$ and marginally for the three-item condition $(Z=1.88 ; p<0.06)$ but not for the two-item condition $(Z=1.38 ; p<0.16)$. Therefore, although we found a relation between BDNF and spatial memory performance as measured by response times, age-related changes in BDNF failed to significantly mediate variation in performance except for the one-item condition.

\section{Mediation effects of hippocampal volume and the association} between BDNF and spatial memory

We ran a series of mediation analyses to determine whether hippocampal volume significantly mediated the association between BDNF and spatial memory performance. In short, we found that volume of the left, but not the right, hippocampus mediated the association between BDNF and spatial memory. We found that the left hippocampus mediated the BDNF association with response times for the one-item condition $(Z=-2.15 ; p<0.03)$ and the two-item condition $(Z=-1.99 ; p<0.04)$ and marginally for the three-item condition $(Z=-1.82 ; p<0.06)$. The left hippocampus also mediated the BDNF association with accuracy rates for the three-item condition $(Z=2.11 ; p<0.03)$ and marginally for the one-item $(Z=1.82 ; p<0.06)$ and two-item $(Z=$ $1.68 ; p<0.09)$ conditions. The volume of the right hippocampus did not mediate the association between BDNF and either response times or accuracy rates. These results indicate that shrinkage of the left hippocampal volume at least partially mediates the BDNF association with memory performance.

\section{Discussion}

The volume of the hippocampus shrinks in late adulthood (Raz et al., 2005; Kennedy et al., 2009), which increases the risk for cognitive impairment (Grundman et al., 2002). However, the molecular factors contributing to hippocampal volume decline in humans has been a matter of speculation. BDNF is critical for 
memory formation and long-term potentiation (Korte et al., 1995; Mu et al., 1999) and is thought to regulate neurogenesis (Benraiss et al., 2001; Lee et al., 2001; Pencea et al., 2001; KatohSemba et al., 2002). In humans, serum and plasma BDNF levels decline with advancing age (Lommatzsch et al., 2005; Ziegenhorn et al., 2007), and genetic studies have identified a single nucleotide polymorphism on the BDNF gene that moderates age-related cognitive decline over a 10 year period (Erickson et al., 2008; Sublette et al., 2008). Given this research, we reasoned that BDNF levels might be associated with age-related hippocampal volume loss. Consistent with this hypothesis, we found that increasing age was associated with reduced levels of BDNF, and reduced levels of BDNF were related to both decline in hippocampal volume and elevated memory deficits.

In rodents, BDNF moderates synaptic plasticity and neurogenesis in the dentate gyrus and has been directly related to learning rates in spatial memory paradigms (Hwang et al., 2006; Rex et al., 2007; Silhol et al., 2007). By blocking either the release of $\mathrm{BDNF}$ or the binding of BDNF to its receptor (TrkB), long-term potentiation is effectively eliminated in the hippocampus (Pang et al., 2004). Furthermore, inducing BDNF production and secretion in the hippocampus can rescue long-term potentiation and relieve spatial memory deficits in aged mice (Rex et al., 2006, 2007; Simmons et al., 2009). In a rodent model of successful aging in which the animals have longer lifespans and preserved memory capacities, BDNF levels were higher than in animals that experience normal age-related patterns of decline (Silhol et al., 2008). BDNF also moderates tau formation (Elliott and Ginzburg, 2006), $\beta$-amyloid neurotoxicity (Arancibia et al., 2008), and hippocampaldependent memory performance in animal models of Alzheimer's disease (Tapia-Arancibia et al., 2008; Blurton-Jones et al., 2009). In summary, BDNF has been convincingly demonstrated to relate to memory formation, neurogenesis, and Alzheimer's disease pathology in aged animals.

In humans, postmortem research has found reduced levels of BDNF in the hippocampus of older adults compared with younger adults and lower levels in individuals with Alzheimer's and Parkinson's diseases compared with age-matched controls (Hock et al., 2000; Murer et al., 2001). Given the challenges associated with measuring BDNF in postmortem tissue, recent studies have examined circulating BDNF in living subjects. The functional significance of BDNF in the blood is a matter of debate, but our results, along with others (Lommatzsch et al., 2005; Ziegenhorn et al., 2007; Gunstad et al., 2008), demonstrate that circulating BDNF levels decline with advancing age. However, the degree to which serum BDNF reflects BDNF levels in the brain (e.g., hippocampus) remains a matter of speculation. Several studies have now reported positive correlations $(r=0.81)$ between serum BDNF and BDNF in both the prefrontal cortex and hippocampus (Karege et al., 2002; Elfving et al., 2009; Sartorius et al., 2009), suggesting that there might be a link between peripheral and central sources of BDNF. BDNF is produced and secreted at several sites in the periphery (e.g., platelets), and, therefore, the results from our study and previous studies could be attributable to parallel actions on peripheral sources and central sources of BDNF and not necessarily attributable to central levels influencing the concentration of BDNF in the periphery. Thus, previous studies on correlations between BDNF in the serum and brain cannot make inferences about the locus of its release (Pan et al., 1998; Sartorius et al., 2009). However, our results build on these previous studies and demonstrate that serum BDNF levels are correlated with measurements of hippocampal volume, an important link that suggests some association between BDNF in the blood and measures of brain integrity
(Lang et al., 2007). Future longitudinal studies should assess the possibility that declining BDNF could be a precursor to cognitive or cortical decay.

Several studies suggest that BDNF levels change rapidly with environmental stimulants such as acute (e.g., $30 \mathrm{~min}$ ) periods of exercise (Gold et al., 2003). Such fluctuation in circulating BDNF levels could influence reliability estimates of serum BDNF. Furthermore, given that we collected blood $\sim 2$ weeks before the neuroimaging session, it is possible that BDNF levels fluctuated across this period. However, our results suggest that any changes in BDNF concentrations across the 2 week period are not enough to eliminate the association with hippocampal volume and memory. Nonetheless, increasing levels of BDNF with exercise suggest that BDNF levels are modifiable. Several studies have found that higher aerobic fitness levels are associated with larger hippocampal volumes (Erickson et al., 2009) and greater volumes of prefrontal and temporal brain regions (Colcombe et al., 2003). It is possible that BDNF plays a critical role in the effects of exercise on the human brain (Kramer and Erickson, 2007).

We found evidence that declining levels of BDNF mediate age-related decline of the left and right hippocampus. Furthermore, BDNF and hippocampal volume mediated spatial memory performance. Interestingly, the mediation results of the hippocampus on age-related memory decline were relatively specific to the left hippocampus and not to the right. Other studies have reported asymmetries in the volume and function of the left and right hippocampus (Erickson et al., 2009) and suggest that the left and right hemispheres might play different, but complementary, roles in memory tasks that emphasize speed. Our results suggest that the left hippocampus is related to measures of speed for all memory set sizes and the right hippocampus only for the threeitem condition.

Although intriguing, these mediation analyses were conducted on cross-sectional data, so it is equally likely that shrinkage of the hippocampus results in lower BDNF levels in the blood. In fact, in another set of mediation analyses, we found that left hippocampal volume mediated the BDNF-spatial memory association. This finding highlights the difficulty of determining the direction of the effects on cross-sectional data. Besides the temporal constraints of interpreting the results from the mediation analysis, other unmeasured third variables correlated with both hippocampal volume and BDNF levels could also influence and explain the mediation results. The only way to formally test for mediation is through a longitudinal study that measures both circulating BDNF levels and hippocampal volumes at multiple time points. Nonetheless, our cross-sectional findings highlight the importance of BDNF as a factor associated with age-related hippocampal volume decay.

We found that circulating levels of BDNF were specific to the volume of the hippocampus and were unrelated to the volume of the caudate nucleus. BDNF is found in the striatum and interacts with the dopaminergic system to regulate parkinsonian symptoms in rodent models (Murer et al., 2001; Collier et al., 2005). Although we failed to find an association between blood levels of BDNF and caudate nucleus volume, we also failed to find any decline in volume of the caudate nucleus with increasing age. This was surprising given that several studies have identified the caudate nucleus as an important site of age-related volume loss (Raz et al., 2005). Our failure to find an age-related decline in caudate nucleus volume might be attributable to the restricted age range of this sample compared with other studies that have reported such effects. Nonetheless, the lack of an association between BDNF and caudate nucleus volume does not suggest that 
BDNF has no relation to the circuitry of the striatum. It is likely that many different molecules other than BDNF are contributing to age-related loss of tissue volume in both the caudate nucleus and the hippocampus. In fact, we found that BDNF only partially mediated the age-related decline in hippocampal volume, indicating that there are other factors besides declining levels of BDNF that contribute to hippocampal decay.

There are several important limitations of our study. First, future studies should examine the association between BDNF and age-related hippocampal volume loss in longitudinal or randomized designs. Longitudinal and randomized trials will help to determine mediation effects and directionality. In addition, our spatial memory findings were only partially related to BDNF levels and only significantly so for response time measures. It will be important for future studies to examine BDNF levels in relation to other hippocampal-dependent tasks that are not as reliant on speeded responses as our task.

In summary, we found an important association between agerelated hippocampal volume loss, decline in spatial memory performance, and reduced levels of circulating BDNF. Given the importance of determining the biomarkers and molecules associated with volume loss in late adulthood, our finding is clinically significant and suggests that interventions that elevate levels of BDNF might help to reduce age-related volume loss. Our mediation analyses, although intriguing, are inherently limited by the cross-sectional nature of the study and the interpretative difficulties with assigning causation to a correlational model. Additional research is necessary to convincingly demonstrate a causal relation between declining levels of BDNF and age-related hippocampal decay. It is also critically important for future studies to identify the potential for healthy lifestyles (e.g., exercise) to moderate BDNF levels in an older adult cohort.

\section{References}

Altar CA, Criden MR, Lindsay RM, DiStefano PS (1993) Characterization and topography of high-affinity 124I-neurotrophin-3 binding to mammalian brain. J Neurosci 13:733-743.

Arancibia S, Silhol M, Moulière F, Meffre J, Höllinger I, Maurice T, TapiaArancibia L (2008) Protective effect of BDNF against beta-amyloid induced neurotoxicity in vitro and in vivo in rats. Neurobiol Dis 31:316-326.

Baron RM, Kenny DA (1986) The moderator-mediator variable distinction in social psychological research: conceptual, strategic, and statistical considerations. J Pers Soc Psychol 51:1173-1182.

Benraiss A, Chmielnicki E, Lerner K, Roh D, Goldman SA (2001) Adenoviral brain-derived neurotrophic factor induces both neostriatal and olfactory neuronal recruitment from endogenous progenitor cells in the adult forebrain. J Neurosci 21:6718-6731.

Blurton-Jones M, Kitazawa M, Martinez-Coria H, Castello NA, Müller FJ, Loring JF, Yamasaki TR, Poon WW, Green KN, LaFerla FM (2009) Neural stem cells improve cognition via BDNF in a transgenic model of Alzheimer's disease. Proc Natl Acad Sci U S A 106:13594-13599.

Bueller JA, Aftab M, Sen S, Gomez-Hassan D, Burmeister M, Zubieta JK (2006) BDNF Val66Met allele is associated with reduced hippocampal volume in healthy subjects. Biol Psychiatry 59:812-815.

Colcombe SJ, Erickson KI, Raz N, Webb AG, Cohen NJ, McAuley E, Kramer AF (2003) Aerobic fitness reduces brain tissue loss in aging humans. J Gerontol A Biol Sci Med Sci 11:342-348.

Collier TJ, Dung Ling Z, Carvey PM, Fletcher-Turner A, Yurek DM, Sladek JR Jr, Kordower JH (2005) Striatal trophic factor activity in aging monkeys with unilateral MPTP-induced parkinsonism. Exp Neurol 191:S60-S67.

Driscoll I, Davatzikos C, An Y, Wu X, Shen D, Kraut M, Resnick SM (2009) Longitudinal pattern of regional brain volume change differentiates normal aging from MCI. Neurology 72:1906-1913.

Egan MF, Kojima M, Callicott JH, Goldberg TE, Kolachana BS, Bertolino A, Zaitsev E, Gold B, Goldman D, Dean M, Lu B, Weinberger DR (2003) The BDNF val66met polymorphism affects activity-dependent secretion of BDNF and human memory and hippocampal function. Cell 112:257-269.
Elfving B, Plougmann PH, Müller HK, Mathé AA, Rosenberg R, Wegener G (2009) Inverse correlation of brain and blood BDNF levels in a genetic rat model of depression. Int J Neuropsychopharmacol 2:1-10.

Elliott E, Ginzburg I (2006) The role of neurotrophins and insulin on tau pathology in Alzheimer's disease. Rev Neurosci 17:635-642.

Erickson KI, Kim JS, Suever BL, Voss MW, Francis BM, Kramer AF (2008) Genetic contributions to age-related decline in executive function: a 10year longitudinal study of COMT and BDNF polymorphisms. Front Hum Neurosci 2:11.

Erickson KI, Prakash RS, Voss MW, Chaddock L, Hu L, Morris KS, White SM, Wójcicki TR, McAuley E, Kramer AF (2009) Aerobic fitness is associated with hippocampal volume in elderly humans. Hippocampus 19: $1030-1039$.

Erickson KI, Voss MW, Prakash RS, Chaddock L, Kramer AF (2010) A cross-sectional study of hormone treatment and hippocampal volume in postmenopausal women: evidence for a limited window of opportunity. Neuropsychology 24:68-76.

Figurov A, Pozzo-Miller LD, Olafsson P, Wang T, Lu B (1996) Regulation of synaptic responses to high-frequency stimulation and LTP by neurotrophins in the hippocampus. Nature 381:706-709.

Fjell AM, Amlien IK, Westlye LT, Stenset V, Fladby T, Skinningsrud A, Eilsertsen DE, Bjørnerud A, Walhovd KB (2010) CSF biomarker pathology correlates with a medial temporo-parietal network affected by very mild to moderate Alzheimer's disease but not a fronto-striatal network affected by healthy aging. Neuroimage 49:1820-1830.

Fukumoto N, Fujii T, Combarros O, Kamboh MI, Tsai SJ, Matsushita S, Nacmias B, Comings DE, Arboleda H, Ingelsson M, Hyman BT, Akatsu H, Grupe A, Nishimura AL, Zatz M, Mattila KM, Rinne J, Goto Y, Asada T, Nakamura S, Kunugi H (2010) Sexually dimorphic effect of the Val66Met polymorphism of BDNF on susceptibility to Alzheimer's disease: new data and meta-analysis. Am J Med Genet B Neuropsychiatr Genet 153B:235-242.

Gold SM, Schulz KH, Hartmann S, Mladek M, Lang UE, Hellweg R, Reer R, Braumann KM, Heesen C (2003) Basal serum levels and reactivity of nerve growth factor and brain-derived neurotrophic factor to standardized acute exercise in multiple sclerosis and controls. J Neuroimmunol 138:99-105.

Greenwood PM, Lambert C, Sunderland T, Parasuraman R (2005) Effects of apolipoprotein E genotype on spatial attention, working memory, and their interaction in healthy, middle-aged adults: results from the National Institute of Mental Health's BIOCARD study. Neuropsychology 19:199-211.

Grundman M, Sencakova D, Jack CR Jr, Petersen RC, Kim HT, Schultz A, Weiner MF, DeCarli C, DeKosky ST, van Dyck C, Thomas RG, Thal LJ (2002) Brain MRI hippocampal volume and prediction of clinical status in a mild cognitive impairment trial. J Mol Neurosci 19:23-27.

Gunstad J, Benitez A, Smith J, Glickman E, Spitznagel MB, Alexander T, Juvancic-Heltzel J, Murray L (2008) Serum brain-derived neurotrophic factor is associated with cognitive function in healthy older adults. J Geriatr Psychiatry Neurol 21:166-170.

Hock CH, Heese K, Olivieri G, Hulette CH, Rosenberg C, Nitsch RM, Otten U (2000) Alterations in neurotrophins and neurotrophin receptors in Alzheimer's disease. J Neural Transm Suppl 59:171-174.

Hwang IK, Yoo KY, Jung BK, Cho JH, Kim DH, Kang TC, Kwon YG, Kim YS, Won MH (2006) Correlations between neuronal loss, decrease of memory, and decrease expression of brain-derived neurotrophic factor in the gerbil hippocampus during normal aging. Exp Neurol 201:75-83.

Judd CM, Kenny DA (1981) Process analysis: estimating mediation in treatment evaluations. Eval Rev 5:602-619.

Kang H, Schuman EM (1995) Long-lasting neurotrophin-induced enhancement of synaptic transmission in the adult hippocampus. Science 267:1658-1662.

Karege F, Schwald M, Cisse M (2002) Postnatal developmental profile of brain-derived neurotrophic factor in rat brain and platelets. Neurosci Lett 328:261-264.

Katoh-Semba R, Asano T, Ueda H, Morishita R, Takeuchi IK, Inaguma Y, Kato K (2002) Riluzole enhances expression of brain-derived neurotrophic factor with consequent proliferation of granule precursor cells in the rat hippocampus. FASEB J 16:1328-1330.

Kawamoto Y, Nakamura S, Nakano S, Oka N, Akiguchi I, Kimura J (1996) Immunohistochemical localization of brain-derived neurotrophic factor in adult rat brain. Neuroscience 74:1209-1226.

Kennedy KM, Erickson KI, Rodrigue KM, Voss MW, Colcombe SJ, Kramer 
AF, Acker JD, Raz N (2009) Age-related differences in regional brain volumes: a comparison of optimized voxel-based morphometry to manual volumetry. Neurobiol Aging 30:1657-1676.

Korte M, Carroll P, Wolf E, Brem G, Thoenen H, Bonhoeffer T (1995) Hippocampal long-term potentiation is impaired in mice lacking brain-derived neurotrophic factor. Proc Natl Acad Sci U S A 92:8856-8860.

Kramer AF, Erickson KI (2007) Capitalizing on cortical plasticity: influence of physical activity on cognition and brain function. Trends Cogn Sci 11: 342-348.

Kukull W, Larson E, Teri L, Bowen J, McCormick W, Pfanschmidt M (1994) The mini-mental state examination score and the clinical diagnosis of dementia. J Clin Epidemiol 47:1061-1067.

Lang UE, Hellweg R, Seifert F, Schubert F, Gallinat J (2007) Correlation between serum brain-derived neurotrophic factor level and an in vivo marker of cortical integrity. Biol Psychiatry 62:530-535.

Lapchak PA, Araujo DM, Beck KD, Finch CE, Johnson SA, Hefti F (1993) BDNF and trkB mRNA expression in the hippocampal formation of aging rats. Neurobiol Aging 14:121-126.

Lee R, Kermani P, Teng KK, Hempstead BL (2001) Regulation of cell survival by secreted proneurotrophins. Science 294:1945-1948.

Lommatzsch M, Zingler D, Schuhbaeck K, Schloetcke K, Zingler C, SchuffWerner P, Virchow JC (2005) The impact of age, weight and gender on BDNF levels in human platelets and plasma. Neurobiol Aging 26:115-123.

MacKinnon DP, Warsi G, Dwyer JH (1995) A simulation study of mediated effect measures. Multivar Behav Res 30:41-62.

MacKinnon DP, Fairchild AJ, Fritz MS (2007) Mediation analysis. Annu Rev Psychol 58:593-614.

Madden DJ, Spaniol J, Whiting WL, Bucur B, Provenzale JM, Cabeza R, White LE, Huettel SA (2007) Adult age differences in the functional neuroanatomy of visual attention: a combined fMRI and DTI study. Neurobiol Aging 28:459-476.

Mu JS, Li WP, Yao ZB, Zhou XF (1999) Deprivation of endogenous brainderived neurotrophic factor results in impairment of spatial learning and memory in adult rats. Brain Res 835:259-265.

Murer MG, Yan Q, Raisman-Vozari R (2001) Brain-derived neurotrophic factor in the control human brain, and in Alzheimer's disease and Parkinson's. Prog Neurobiol 63:71-124.

Ozan E, Okur H, Eker C, Eker OD, Gönül AS, Akarsu N (2010) The effect of depression, BDNF gene val66met polymorphism and gender on serum BDNF levels. Brain Res Bull 81:61-65.

Pan W, Banks WA, Fasold MB, Bluth J, Kastin AJ (1998) Transport of brainderived neurotrophic factor across the blood-brain barrier. Neuropharmacology 37:1553-1561.

Pang PT, Teng HK, Zaitsev E, Woo NT, Sakata K, Zhen S, Teng KK, Yung WH, Hempstead BL, Lu B (2004) Cleavage of proBDNF by tPA/plasmin is essential for long-term hippocampal plasticity. Science 306:487-491.

Patenaude B, Smith SM, Kennedy D, Jenkinson M (2007a) FIRST: FMRIB's integrated registration and segmentation tool. Presented at the 13th Annual Meeting of the Organization for Human Brain Mapping, Chicago, June $10-14$.

Patenaude B, Smith SM, Kennedy D, Jenkinson M (2007b) Bayesian shape and appearance models. Technical report TR07BP1. Oxford: FMRIB Centre, University of Oxford.

Pencea V, Bingaman KD, Wiegand SJ, Luskin MB (2001) Infusion of brainderived neurotrophic factor into the lateral ventricle of the adult rat leads to new neurons in the parenchyma of the striatum, septum, thalamus, and hypothalamus. J Neurosci 21:6706-6717.

Pezawas L, Verchinski BA, Mattay VS, Callicott JH, Kolachana BS, Straub RE, Egan MF, Meyer-Lindenberg A, Weinberger DR (2004) The brainderived neurotrophic factor val66met polymorphism and variation in human cortical morphology. J Neurosci 24:10099-10102.

Phillips HS, Hains JM, Laramee GR, Rosenthal A, Winslow JW (1990) Widespread expression of BDNF but not NT3 by target areas of basal forebrain cholinergic neurons. Science 250:290-294.

Raz N, Lindenberger U, Rodrigue KM, Kennedy KM, Head D, Williamson A, Dahle C, Gerstorf D, Acker JD (2005) Regional brain changes in aging healthy adults: general trends, individual differences and modifiers. Cereb Cortex 15:1676-1689.

Rex CS, Lauterborn JC, Lin CY, Kramár EA, Rogers GA, Gall CM, Lynch G (2006) Restoration of long-term potentiation in middle-aged hippocampus after induction of brain-derived neurotrophic factor. J Neurophysiol 96:677-685.
Rex CS, Lin CY, Kramár EA, Chen LY, Gall CM, Lynch G (2007) Brainderived neurotrophic factor promotes long-term potentiation-related cytoskeletal changes in adult hippocampus. J Neurosci 27:3017-3029.

Salthouse TA, Atkinson TM, Berish DE (2003) Executive functioning as a potential mediator of age-related cognitive decline in normal adults. J Exp Psychol Gen 132:566-594.

Sartorius A, Hellweg R, Litzke J, Vogt M, Dormann C, Vollmayr B, DankerHopfe H, Gass P (2009) Correlations and discrepancies between serum and brain tissue levels of neurotrophins after electroconvulsive treatment in rats. Pharmacopsychiatry 42:270-276.

Schmidt-Kastner R, Wetmore C, Olson L (1996) Comparative study of brain-derived neurotrophic factor messenger RNA and protein at the cellular level suggests multiple roles in hippocampus, striatum, and cortex. Neuroscience 74:161-183.

Silhol M, Arancibia S, Maurice T, Tapia-Arancibia L (2007) Spatial memory training modifies the expression of brain-derived neurotrophic factor tyrosine kinase receptors in young and aged rats. Neuroscience 146:962-973.

Silhol M, Arancibia S, Perrin D, Maurice T, Alliot J, Tapia-Arancibia L (2008) Effect of aging on brain-derived neurotrophic factor, proBDNF, and their receptors in the hippocampus of Lou/C rats. Rejuvenation Res 11:1031-1040.

Simmons DA, Rex CS, Palmer L, Pandyarajan V, Fedulov V, Gall CM, Lynch G (2009) Up-regulating BDNF with an ampakine rescues synaptic plasticity and memory in Huntington's disease knockin mice. Proc Natl Acad Sci U S A 106:4906-4911.

Smith SM, Jenkinson M, Woolrich MW, Beckmann CF, Behrens TE, JohansenBerg H, Bannister PR, De Luca M, Drobnjak I, Flitney DE, Niazy RK, Saunders J, Vickers J, Zhang Y, De Stefano N, Brady JM, Matthews PM (2004) Advances in functional and structural $M R$ image analysis and implementation as FSL. Neuroimage 23 [Suppl 1]:S208-S219.

Sobel ME (1982) Asymptotic confidence intervals for indirect effects in structural equation models. Sociol Methodol 13:290-312.

Stern Y, Sano M, Paulson J, Mayeau R (1987) Modified mini-mental state examination: validity and reliability. Neurology 37:179.

Stoop R, Poo MM (1996) Synaptic modulation by neurotrophic factors: differential and synergistic effects of brain-derived neurotrophic factor and ciliary neurotrophic factor. J Neurosci 16:3256-3264.

Sublette ME, Baca-Garcia E, Parsey RV, Oquendo MA, Rodrigues SM, Galfalvy H, Huang YY, Arango V, Mann JJ (2008) Effect of BDNF val66met polymorphism on age-related amygdala volume changes in healthy subjects. Prog Neuropsychopharmacol Biol Psychiatry 32: 1652-1655.

Szeszko PR, Lipsky R, Mentschel C, Robinson D, Gunduz-Bruce H, Sevy S, Ashtari M, Napolitano B, Bilder RM, Kane JM, Goldman D, Malhotra AK (2005) Brain-derived neurotrophic factor val66met polymorphism and volume of the hippocampal formation. Mol Psychiatry 10:631-636.

Takahashi J, Palmer TD, Gage FH (1999) Retinoic acid and neurotrophins collaborate to regulate neurogenesis in adult-derived neural stem cell cultures. J Neurobiol 38:65-81.

Tanaka J, Horiike Y, Matsuzaki M, Miyazaki T, Ellis-Davies GC, Kasai H (2008) Protein synthesis and neurotrophin-dependent structural plasticity of single dendritic spines. Science 319:1683-1687.

Tapia-Arancibia L, Aliaga E, Silhol M, Arancibia S (2008) New insights into brain BDNF function in normal aging and Alzheimer's disease. Brain Res Rev 59:201-220.

Walhovd KB, Westlye LT, Amlien I, Espeseth T, Reinvang I, Raz N, Agartz I, Salat DH, Greve DN, Fischl B, Dale AM, Fjell AM (2009) Consistent neuroanatomical age-related volume differences across multiple samples. Neurobiol Aging. Advance online publication. Retrieved June 29, 2009. doi:10.1016/j.neurobiolaging.2009.05.013.

Wetmore C, Ernfors P, Persson H, Olson L (1990) Localization of brainderived neurotrophic factor mRNA to neurons in the brain by in situ hybridization. Exp Neurol 109:141-152.

Zhang Y, Brady M, Smith S (2001) Segmentation of brain MR images through a hidden Markov random field model and expectation maximization algorithm. IEEE Trans Med Imaging 20:45-57.

Ziegenhorn AA, Schulte-Herbrüggen O, Danker-Hopfe H, Malbranc M, Hartung HD, Anders D, Lang UE, Steinhagen-Thiessen E, Schaub RT, Hellweg R (2007) Serum neurotrophins: a study on the time course and influencing factors in a large old age sample. Neurobiol Aging 28:1436-1445. 